\title{
Fatores associados a infecções puerperais na maternidade de um hospital escola do interior de São Paulo
}

Factors associated with puerperal infection in the maternity unit of a hospital school in São Paulo

\begin{abstract}
RESUMO
Objetivos: A pesquisa teve como objetivo identificar os fatores associados à infecção puerperal em puérperas de janeiro de 2017 a janeiro de 2019; relacionar a infecção puerperal com as variáveis sociodemográficas e clínicas; relacionar via de parto com a infecção puerperal. Métodos: O local do estudo foi uma maternidade do interior do estado de São Paulo. É um estudo exploratório, quantitativo e retrospectivo, no qual foi utilizado o teste de $\chi^{2} \mathrm{o}$ ao nível de significância de $5 \%$. A coleta de dados aconteceu de forma exclusiva em prontuários, após a aprovação pelo Comitê de Ética e Pesquisa, por meio do parecer $\mathrm{n}^{\circ} 3.144 .647$. Resultados: $\mathrm{O}$ grupo de puérperas estudado $(\mathrm{N}=20)$ caracteriza-se por adultas jovens, em união estável, com ensino médio completo, do lar e dependentes do Sistema Único de Saúde. As variáveis que apresentaram dependência foram a relação entre classificação de ferida operatória e escolaridade $(p=0,035)$; a classificação de sinais e sintomas e o estado civil; e a classificação do plano terapêutico e a reabordagem da ferida operatória $(\mathrm{p}=0,067)$. Foram evidenciados, como antecedentes clínicos predominantes, o tabagismo, a asma e a hipertensão arterial sistêmica. Quanto às condições de parto, este foi marcado por tentativa de indução, toques vaginais frequentes e desfecho de cesariana. Conclusão: Foi constatada a relação entre parto cesáreo e infecção puerperal, ressaltando o procedimento invasivo e maior incidência de casos de sepse puerperal após cesariana.
\end{abstract}

Palavras-chave: infecção puerperal; infecção hospitalar; parto normal; cesárea; enfermagem obstétrica.

\begin{abstract}
Objective: This study aimed to identify the factors associated with puerperal infection in postpartum women from January 2017 to January 2019; to associate puerperal infection with socio-demographic variables; and to associate delivery mode with puerperal infection. Method: The locus of study was the maternity unit of a hospital in the state of São Paulo. This was an exploratory, quantitative and retrospective study which employed the $\chi^{2}$ test at a 5\% significance level. Data collection took place exclusively in medical records after approval by the Ethics and Research Committee through opinion n. 3,144,647. Results: The postpartum group studied was composed of young adult housewives, in domestic partnership, with completed high school education, who rely on the Unified Health System. The variables that showed dependence were: surgical wound classification and education $(\mathrm{p}=0.035)$; the classification of signs and symptoms and marital status; and the classification of the therapeutic plan and the revaluation of the surgical wound $(\mathrm{p}=0.067)$. The predominant clinical antecedents were smoking, asthma and systemic arterial hypertension. As for delivery conditions, they was marked by attempted induction, frequent vaginal touches and cesarean outcome. Conclusion: The results reinforce the relation between cesarean section and puerperal infection, showing evidence of invasive procedure and higher incidence of cases of puerperal sepsis after cesarean section.

Descriptors: puerperal infections; cross infection; natural childbirth; cesarean section; obstetrical nursing.
\end{abstract}

${ }^{1}$ Pontifícia Universidade Católica de São Paulo, Faculdade de Ciências Médicas e da Saúde - Sorocaba (SP), Brasil. Autora correspondente: Janie Maria de Almeida - Pontifícia Universidade Católica de São Paulo, Faculdade de Ciências Médicas e da Saúde - Rua Joubert Wey, 290 - CEP: 18030-070 - Sorocaba (SP), Brasil - E-mail: janie@ pucsp.br Recebido em 26/11/2019. Aceito para publicação em 26/07/2021. 


\section{INTRODUÇÃO}

As infecções associadas aos cuidados de saúde são um problema envolvido na temática da segurança do paciente, a qual está inserida no Programa Nacional da Segurança do Paciente (PNSP) do Ministério da Saúde, visando à promoção de medidas que reduzam riscos ao indivíduo no ambiente hospitalar. ${ }^{1}$

A infecção puerperal, conhecida também como febre puerperal, teve a sua primeira descrição no século V a.C., por Hipócrates. ${ }^{2}$

Segundo o Ministério da Saúde, ela

caracteriza-se por qualquer infecção do trato genital ocorrida durante o puerpério. Entre outras manifestações, a paciente pode apresentar a febre puerperal, conceituada por temperatura axilar maior ou igual a $38^{\circ} \mathrm{C}$ manifestada após 24 horas do parto com duração mínima de dois dias. ${ }^{3}$

As prevalências de infecção puerperal nos países da Europa oscilam entre 3 e 20\%, com valores médios de $9 \%$. No Brasil, ainda existem poucos dados sobre sepse puerperal, que são baseados em estudos descritivos de alguns poucos centros isolados ${ }^{4}$. Segundo Gabriellone e Barbieri, o valor no Brasil varia de 1 a $7,2 \% .^{4}$

A cesariana pode ser considerada um fator predisponente à infecção puerperal, mais precisamente a infecção do sítio cirúrgico, ${ }^{5}$ visto que apresenta risco aumentado de até $1,5 \mathrm{vez}$ a mais em relação ao parto vaginal. ${ }^{6}$ Nesse quesito, o Brasil tem uma das taxas mais elevadas de cesariana no mundo, e esse procedimento correspondeu no ano de 2015 a 55\% dos partos no país. ${ }^{7}$ Confrontando-se com esse valor, a Organização Mundial da Saúde (OMS) preconiza que o total de partos cesáreos em uma instituição de saúde seja de até 15\% para a preservação da saúde materna e/ou fetal. ${ }^{8}$

O estudo teve como objetivo verificar os fatores associados à infecção puerperal, relacionando-a com as variáveis sociodemográficas e o parto cesáreo.

\section{MATERIAIS E MÉTODOS}

Trata-se de um estudo exploratório, quantitativo e retrospectivo. Foi estudada uma amostra $(\mathrm{N}=20)$ de puérperas que apresentaram casos de infecção puerperal durante a internação. Os critérios de inclusão para o trabalho foram prontuários de puérperas maiores de 18 anos que deram à luz na maternidade do interior do estado de São Paulo e que tiveram infecção puerperal, no período janeiro de 2017 a janeiro de 2019. Os critérios de exclusão foram os prontuários das mulheres que não apresentaram o quadro clínico de infecção puerperal, os que traziam dados incompletos ou inconclusivos e os de pacientes menores de 18 anos.

Para a análise dos resultados foi empregada a estatística descritiva e inferencial. Para verificar a associação entre as variáveis preditoras e o evento infecção puerperal, utilizou-se o teste $\chi^{2}$ ao nível de significância de $5 \%$.
A pesquisa foi realizada respeitando os princípios éticos definidos na resolução 466/2012. A coleta de dados foi iniciada após a aprovação do Comitê de Ética e Pesquisa (CEP), por meio do parecer $n^{\circ} 3.144 .647$. Após a aprovação, foi empregado o Termo de Compromisso de Confidencialidade para a realização da coleta de dados de forma única e exclusiva nos prontuários.

\section{RESULTADOS E DISCUSSÃO}

Este estudo teve como objetivos identificar os fatores associados à infecção puerperal em uma maternidade do interior do estado de São Paulo, associar as variáveis sociodemográficas, obstétricas e clínicas à infecção puerperal e relacionar a via de parto com esse evento.

As associações foram realizadas entre os aspectos sociodemográficos, compostos das variáveis idade, estado civil, escolaridade, ocupação e plano de saúde, e a classificação da ferida operatória, dividida em leve, moderada e grave. Foi analisada a dependência entre esses dois fatores, conforme exposto na Tabela 1.

O grupo de puérperas pesquisado caracteriza-se por adultas jovens, em união estável, com ensino médio completo, que são do lar e dependentes do SUS.

As variáveis idade, estado civil, ocupação e plano de saúde não apresentaram dependência com a infecção puerperal.

A variável escolaridade apresentou nível de dependência com a classificação da ferida operatória, ou seja, predominaram mulheres com ensino médio completo e ferida do tipo moderada, com $\mathrm{p}=0,035$.

A gravidade da infecção puerperal pode estar interligada com as condições sociais da pessoa, uma vez que esses aspectos podem estar relacionados com menor nível de instrução, condições de higiene e moradia. ${ }^{9}$

As associações realizadas entre os dados obstétricos compostos das variáveis idade gestacional, número de consultas de pré-natal, tipo de parto, dados da evolução do trabalho de parto, número de toques e condutas durante o parto - e a classificação da ferida operatória para avaliar a dependência entre esses fatores estão expostas na Tabela 2.

Este grupo de mulheres apresentou gestação a termo, mais de sete consultas de pré-natal, predomínio de cesárea, parto marcado por indução com hormônios sintéticos, com duração menor que 6 horas, com um a cinco toques vaginais durante o trabalho de parto e maior número de condutas. O uso de antibióticos esteve associado a anti-inflamatórios no procedimento da cesárea; especificamente, de acordo com registros do prontuário, a infusão de antibióticos tinha objetivo profilático, ou seja, evitar possíveis infecções e destruir bactérias que pudessem se alojar no útero durante a cesariana.

Quanto aos itens evolução de trabalho de parto e número de toques vaginais, eles apresentaram diferença na amostra estudada, tendo sido evidenciados, respectivamente, por 15 e 18 puérperas. Isso ocorreu uma vez que três prontuários 
Tabela 1. Associação entre os dados sociodemográficos e a classificação da ferida operatória em puérperas internadas em uma maternidade do interior de São Paulo, 2017 a 2019, Sorocaba/SP.

\begin{tabular}{|c|c|c|c|c|c|}
\hline \multirow{2}{*}{ Variável } & \multicolumn{3}{|c|}{ Classificação da ferida operatória } & \multirow{2}{*}{ Total } & \multirow{2}{*}{ p-valor } \\
\hline & Leve & Moderada & Grave & & \\
\hline \multicolumn{6}{|l|}{ 1. Idade (anos) } \\
\hline 20 a 25 & 1 & 5 & 0 & 6 & \multirow{4}{*}{0,915} \\
\hline 26 a 30 & 1 & 1 & 1 & 3 & \\
\hline 31 a 35 & 1 & 3 & 2 & 6 & \\
\hline Mais de 35 & 1 & 2 & 1 & 4 & \\
\hline \multicolumn{6}{|l|}{ 2. Estado civil } \\
\hline Divorciada & 0 & 1 & 0 & 1 & \multirow{3}{*}{0,862} \\
\hline Solteira & 1 & 2 & 2 & 5 & \\
\hline União estável & 3 & 9 & 2 & 14 & \\
\hline \multicolumn{6}{|l|}{ 3. Escolaridade } \\
\hline Ensino fundamental incompleto & 0 & 2 & 1 & 3 & \multirow{6}{*}{0,035} \\
\hline Ensino médio completo & 0 & 9 & 2 & 11 & \\
\hline Ensino médio incompleto & 2 & 0 & 1 & 3 & \\
\hline Ensino superior completo & 1 & 0 & 0 & 1 & \\
\hline Ensino superior incompleto & 0 & 1 & 0 & 1 & \\
\hline Sem Escolaridade & 1 & 0 & 0 & 1 & \\
\hline \multicolumn{6}{|l|}{ 4. Ocupação } \\
\hline Autônomo & 0 & 2 & 1 & 3 & \multirow{4}{*}{0,757} \\
\hline Desempregada & 0 & 1 & 0 & 1 & \\
\hline Do lar & 2 & 8 & 2 & 12 & \\
\hline Empregada & 2 & 1 & 1 & 4 & \\
\hline \multicolumn{6}{|l|}{ 5. Plano de Saúde } \\
\hline Convênio & 1 & 1 & 0 & 2 & \multirow{2}{*}{1,000} \\
\hline Sistema Único de Saúde & 3 & 11 & 4 & 18 & \\
\hline
\end{tabular}

não continham dados do uso de medicamentos para a indução do parto.

Segundo Downe et al., ${ }^{10}$

o toque realizado em intervalos regulares, sozinho ou como parte do partograma (um gráfico pré-impresso que fornece uma visão geral da evolução do trabalho de parto), visa avaliar se o trabalho de parto está evoluindo de forma fisiológica, e detectar precocemente os casos que estão evoluindo lentamente.

O toque é recomendado na admissão da paciente para a avaliação do colo uterino e, na fase ativa do trabalho de parto, pode ser realizado de $4 \mathrm{em} 4$ horas e de acordo com o desejo da mulher. ${ }^{11}$
O local pesquisado é um hospital escola onde se encontram estagiários e residentes de Medicina. A realização de elevado número de toques vaginais tem sido constatada principalmente nesses locais, nos quais são desenvolvidas atividades para a formação de profissionais da saúde, enfermeiros e médicos, situação esta corroborada pelo trabalho de Diniz et al. ${ }^{12}$

O toque vaginal obteve como resultado a predominância de um a cinco toques durante o trabalho de parto, de acordo com os registros em prontuário. Entretanto, os registros da equipe de enfermagem e médica não são fidedignos uma vez que, na prática, o número de toques é mais frequente. Esse fato torna-se preocupante visto que os registros são uma forma de comunicação dos cuidados prestados pelos profissionais durante a assistência. ${ }^{12}$ Sabe-se que, durante o toque, 
Tabela 2. Associação entre os dados obstétricos e a classificação da ferida operatória em uma maternidade do interior de São Paulo, 2017 a 2019, Sorocaba/SP.

\begin{tabular}{|c|c|c|c|c|c|}
\hline \multirow{2}{*}{ Variável } & \multicolumn{3}{|c|}{ Classificação da ferida operatória } & \multirow{2}{*}{ Total } & \multirow{2}{*}{ p-valor } \\
\hline & Leve & Moderada & Grave & & \\
\hline \multicolumn{6}{|l|}{ 1. Idade gestacional (semanas) } \\
\hline 37 a 42 & 3 & 12 & 4 & 19 & \multirow{2}{*}{0,408} \\
\hline Menos de 37 & 1 & 0 & 0 & 1 & \\
\hline \multicolumn{6}{|l|}{ 2. Número de consultas pré-natal } \\
\hline 1 a 6 & 1 & 4 & 2 & 7 & \multirow{2}{*}{1,000} \\
\hline Mais de 7 & 3 & 8 & 2 & 13 & \\
\hline
\end{tabular}

\section{Parto}

$\begin{array}{lccccc}\text { Cesárea } & 2 & 11 & 3 & 16 & \\ \text { Fórceps } & 1 & 0 & 0 & 1 & 0,414 \\ \text { Normal } & 1 & 1 & 1 & 3 & \end{array}$

\section{Evolução do trabalho de parto}

Sem indução
Uso de indução (Misoprostol)
Uso de indução (Ocitocina)

\section{Duração do parto}

Duração entre 7 e 12 horas

Duração maior que 13 horas

Duração menor que 6 horas

$\begin{array}{lllcl}2 & 7 & 1 & 10 & \\ 0 & 0 & 1 & 1 & 0,321 \\ 2 & 4 & 1 & 7 & \end{array}$

\section{Número de toques vaginais}

1 a 5
6 ou mais
Nenhum

\section{Condições no parto}

Episiotomia, uso de antibiótico profilático

Períneo íntegro

Períneo íntegro, uso de antibiótico profilático

Períneo íntegro, uso de antibiótico profilático, uso de AINE

Períneo lacerado, uso de AINE

Uso de AINE

Uso de antibiótico profilático

Uso de antibiótico profilático, uso de AINE

$\begin{array}{llll}1 & 2 & 2 & 5 \\ 1 & 1 & 0 & 2 \\ 2 & 9 & 2 & 13\end{array}$

5
2

13

$\begin{array}{ccccc}4 & 3 & 6 & 13 & \\ 1 & 0 & 0 & 1 & 0,801 \\ 0 & 0 & 1 & 1 & \end{array}$

$\begin{array}{lllll}1 & 0 & 0 & 1 & \\ 0 & 0 & 1 & 1 & \\ 0 & 0 & 2 & 2 & \\ 1 & 2 & 0 & 3 & 0,109 \\ 0 & 1 & 0 & 1 & \\ 0 & 1 & 0 & 1 & \\ 0 & 2 & 0 & 2 & \\ 2 & 6 & 1 & 9\end{array}$

sem.: semanas; AINE: anti-inflamatórios não esteroides.

há riscos envolvidos em razão do contato com o canal vaginal, que apresenta microbiota própria. Dessa forma, o descuido com esse tipo de exame facilita a aquisição de uma infecção pós-cesariana. ${ }^{13}$

O toque é uma prática realizada para o acompanhamento do processo fisiológico do parto, devendo ser feito por médicos e enfermeiras obstétricas com a frequência preconizada pelo Ministério da Saúde, de um toque a cada 4 horas, em média, na fase ativa do trabalho de parto, garantindo informações relativas à espessura do colo uterino e à apresentação fetal, de acordo com o desejo da parturiente. Dessa forma, é possível assegurar um parto humanizado, ou seja, que respeite os direitos da mulher e seja respaldado técnica e cientificamente. ${ }^{12}$

Além dos riscos citados acima, um número excessivo de toques vaginais pode ser compreendido como abuso e violência contra a mulher, dada a realização de um procedimento tão íntimo, muitas vezes, sem o consentimento dela. Foi realizado um seminário na Faculdade de Saúde Pública da Universidade de São Paulo, no qual foi discutido o tema "vagina-escola" abordando-se a concepção de que os profissionais médicos e enfermeiros têm o poder de acessar livremente o corpo das mulheres. Essa ideia deve ser descontruída 
e desnaturalizada, promovendo a proteção à saúde da mulher e o direito de a parturiente ter autonomia durante o parto. ${ }^{11}$

Uma das formas de auxiliar o exame da dilatação cervical, segundo a literatura, é pela avaliação da "linha púrpura" durante da fase ativa do trabalho de parto, o que evita traumas e reações indesejadas pelas mulheres. Esse método consiste na avaliação da coloração arroxeada que surge na região perianal da mulher formando uma linha ascendente da fissura interglútea (ânus) em direção sacrococcígea e atingindo $10 \mathrm{~cm}$ de comprimento, o que indica dilatação completa. Isso ocorre uma vez que a descida da apresentação fetal na bacia da mulher provoca uma prensa no tecido do sacro, causando vasocongestão e descoloração local. ${ }^{14}$

O tipo de parto predominante foi o parto cesáreo, considerado um dos fatores mais importantes para o desenvolvimento da sepse puerperal. De acordo com Lima et al. ${ }^{15}$ a cesárea aumenta de cinco a 30 vezes a chance de se desenvolver infecção puerperal. Isso ocorre por diversos motivos, sendo os principais a incisão cirúrgica, a maior perda sanguínea e o procedimento invasivo, de modo que a paciente fica mais exposta a contrair diversas bactérias. Contradizendo o que é preconizado pela OMS, isto é, a taxa máxima de $15 \%$, o índice de partos cesáreos mantém-se elevado no país.

As associações a seguir foram realizadas com os dados da ferida operatória, que incluem as variáveis reabordagem e ferida operatória, para analisar a dependência entre esses fatores, como exposto na Tabela 3.

Quanto às variáveis da ferida operatória e os sinais e sintomas, eles não apresentaram dependência com a infecção puerperal. A variável reabordagem da ferida operatória apresentou dependência com a infecção puerperal, demonstrada por $\mathrm{p}=0,067$.

Foi esperada a relação de dependência entre a classificação do plano terapêutico e a reabordagem da ferida operatória uma vez que, quando se apresenta esta última, o plano terapêutico é mais complexo em virtude do procedimento invasivo, o qual exige maior necessidade de cuidados médicos e de enfermagem.

Tabela 3. Associação entre os dados de tratamento da ferida operatória e a classificação do plano terapêutico em uma maternidade do interior de São Paulo, 2017 a 2019, Sorocaba/SP.

\begin{tabular}{lccc} 
Variável & \multicolumn{2}{c}{ Classificação do plano terapêutico } & Total p-valor \\
& Leve Moderado Grave
\end{tabular}

\section{Reabordagem da ferida operatória}

Não

Sim

\section{Ferida operatória}

Deiscência, dor

Deiscência, dor, secreção serosa

Deiscência, hiperemiada, dor, secreção serossanguinolenta

Deiscência, hiperemiada, secreção serosa

Deiscência, hiperemiada, secreção serosa, edema, endurecimento

Deiscência, secreção serossanguinolenta

Dor, secreção sanguinolenta

Dor, secreção serosa

Dor, secreção serosa, secreção sanguinolenta, secreção

serosa

Hiperemiada, dor

Hiperemiada, dor, edema

Hiperemiada, dor, secreção acastanhada

Hiperemiada, dor, secreção serossanguinolenta

Hiperemiada, secreção serossanguinolenta

Hiperemiada, úmida, secreção serosa

Secreção sanguinolenta, sangramento fétido

Secreção serosa, secreção serossanguinolenta

$\begin{array}{ll}5 & 6 \\ 1 & 0\end{array}$

0

$\begin{array}{cc}2 & 13 \\ 4 & 5\end{array}$

0,067

0

0

0

1

1

1

0

0

0
1
0
0

1

1

0

1

0

0

1

0

0

1

0

0

1

0

1

0

0

0

1

0

0

1

0

0

0

1

0

0

0

0

1

0
0,088

2

1

1

2

1

1

1

1

Continua... 
Tabela 3. Continuação.

\title{
Variável
}

Classificação do plano terapêutico

Leve

Moderado

Grave

\section{Sinais e sintomas}

Dor abdominal

Dor abdominal, lóquios fétidos

0

Hipoinvolução uterina

0

Lóquios fétidos

Temperatura axilar igual ou maior que $38^{\circ} \mathrm{C}$

Temperatura axilar igual ou maior que $38^{\circ} \mathrm{C}$,

Dor abdominal

Temperatura axilar igual ou maior que $38^{\circ} \mathrm{C}$,

Dor abdominal, lóquios fétidos

Temperatura axilar igual ou maior que $38^{\circ} \mathrm{C}$,

Dor abdominal

$\begin{array}{llll}2 & 0 & 3 & 5 \\ 0 & 2 & 0 & 2 \\ 0 & 1 & 0 & 1 \\ 2 & 0 & 0 & 2 \\ 0 & 1 & 1 & 2 \\ 0 & 2 & 1 & 3 \\ 0 & 1 & 0 & 1 \\ 1 & & & \\ & & & 1\end{array}$

\author{
5 \\ 2 \\ 1 \\ 2 \\ 2 \\ 3
}

0,173

Do grupo pesquisado de 20 puérperas, 18 prontuários apresentaram anotações sobre a reabordagem da ferida operatória.

O procedimento pode causar prejuízos para a mulher, que sofre alterações psicológicas e emocionais em decorrência do quadro clínico, e o vínculo entre mãe e filho é interrompido. Consequentemente, compromete-se o futuro reprodutivo da puérpera. ${ }^{11}$ Nesse caso, há também impactos para o hospital já que aumentam os gastos financeiros com materiais, medicamentos e honorários médicos. Além disso, pode ocorrer um desfecho mais grave: o óbito materno.

É fundamental ressaltar que a ferida operatória é um processo dinâmico, devendo ser avaliada diariamente por meio do exame físico para acompanhamento e evolução e podendo relacionar-se com o processo fisiológico da cicatrização. Além disso, devem-se seguir prescrições de curativos adequadas para o sucesso no tratamento. ${ }^{15,16}$

Todas essas questões estão envolvidas no parto cesáreo, que é o fator determinante para o maior número de reabordagens de ferida operatória e, consequentemente, de tratamentos mais longos e prejudiciais para a paciente e para o hospital. ${ }^{15}$

\section{CONCLUSÕES}

Com base no estudo realizado, foi constatada a dependência estatística do parto cesáreo com a infecção puerperal. As variáveis que apresentaram dependência foram: ferida operatória com escolaridade, sinais e sintomas com estado civil e plano terapêutico com reabordagem da ferida operatória.
A infecção puerperal é um processo que pode gerar diversas complicações para a saúde materna e também pode colocar em risco a vida da paciente, contribuindo para o aumento da taxa de morte materna.

Este estudo reafirmou a necessidade de apontar caminhos para que sejam implantadas medidas que reduzam os casos de parto cesáreo, assegurando, acima de tudo, um parto seguro e com riscos mínimos de infecção puerperal.

\section{Conflitos de interesse}

Os autores declaram inexistência de conflito de interesses na realização deste trabalho.

\section{REFERÊNCIAS}

1. Pina E, Ferreira E, Masques A, Matos B. Infecções associadas aos cuidados de saúde e segurança do doente. Rev Port Saúde Pública. 2010;(10):27-39.

2. Berlet LJ. Infecção no período puerperal: implicações para a enfermagem [dissertação]. Rio de Janeiro: Universidade do Estado do Rio de Janeiro; 2015.

3. Brasil. Agência Nacional de Vigilância Sanitária. Medidas de prevenção e critérios diagnósticos de infecções puerperais em parto vaginal e cirurgia cesariana. Brasília: Anvisa; 2017.

4. Gabrielloni MC, Barbieri M. Infecção hospitalar e suas interfaces na área de saúde. In: Fernandes AT, Fernandes MOV, Ribeiro N, eds. Infecção hospitalar e suas interfaces na área de saúde. São Paulo: Atheneu; 2002. p. 91-128. 
5. Karsnitz DB. Puerperal infections of the genital tract: a clinical review. J Midwifery Women's Health. 2013;58(6):632-42. https://doi.org/10.1111/jmwh.12119

6. Petter CE, Farret TCF, Scherer JS, Antonello VS. Fatores relacionados a infecções de sítio cirúrgico após procedimentos obstétricos. Sci Med. 2013;23(1):28-33. https://doi.org/10.15448/1980-6108.2013.1.12715

7. Cunha MR Padoveze MC, Melo CRM, Nichiata LYI. Identificação da infecção de sítio cirúrgico pós-cesariana: consulta de enfermagem. Rev Bras Enferm. 2018;71(supl. 3):1395-403. https://doi. org/10.1590/0034-7167-2017-0325

8. Brasil. Ministério da Saúde. CONITEC. Diretrizes de Atenção a Gestante: a operação cesariana. Brasília: Ministério da Saúde; 2015.

9. Benincasa BC, Walker C, Cioba C, Rosa CCS, Martins DE, Dias E, et al. Taxas de infecção relacionadas a parto cesáreos e normais no Hospital de Clínicas de Porto Alegre. Rev HCPA. 2012;32(1):5-9.

10. Downe S, Gyte GML, Dahlen HG, Singata M. Toque vaginal de rotina para avaliar a evolução do trabalho de parto e melhorar desfechos maternos e de bebês a termo. Cochrane Data System Rev. 2013. https://doi. org/10.1002/14651858.CD010088.pub2

11. Brasil. Ministério da Saúde. Secretaria de Ciência, Tecnologia e Insumos Estratégicos. Departamento de Gestão e Incorporação de Tecnologias em Saúde. Diretrizes nacionais de assistência ao parto normal: versão resumida. Brasília: Ministério da Saúde; 2017.

12. Diniz CSG, Niy DY, Andrezzo HFA, Carvalho PCA, Salgado HO. A vagina-escola: seminário interdisciplinar sobre violência contra a mulher no ensino das profissões de saúde. Interface (Botucatu). 2016;20(56):253-9. https://doi.org/10.1590/1807-57622015.0736

13. Ataide MM, Santos AAP, Silva JMO, Sanches METL. Exame obstétrico realizado pela enfermeira: da teoria a prática. Enferm Foco. 2016;7(2):67-71. https://doi. org/10.21675/2357-707X.2016.v7.n2.798

14. Maranhão AMSA, Barbieri M, Gabrielloni MC, Fustinoni SM. Cuidados na realização da propedêutica obstétrica visando a prevenção da infecção hospitalar em maternidades. Rev Bras Enferm. 1992;45(4):302-7. https://doi.org/10.1590/ S0034-71671992000300008

15. Lima DM, Wall ML, Hey A, Falcade AC, Chaves ACM, Souza MAR. Fatores de riscos para infecção no puerpério cirúrgico. Cogitare Enferm. 2014;19(4):73440. http://dx.doi.org/10.5380/ce.v19i4.35170

16. Silva CG, Crossetti MGO. Curativos para tratamento de feridas operatórias abdominais: uma revisão sistemática. Rev Gaúcha Enferm. 2012;33(3):182-9. https://doi.org/10.1590/S1983-14472012000300024

\section{Como citar este artigo:}

Almeida JM, Demizu NTL, Oliveira MR. Fatores associados a infecções puerperais na maternidade de um hospital escola do interior de São Paulo. Rev Fac Ciênc Méd Sorocaba. 2020;22(3):112-8. http://doi.org/10.23925/1984-4840.2020v22i3a5 